\title{
Variations in knowledge, awareness and treatment of hypertension and stroke risk by country income level
}

\author{
Martin O' Donnell, ${ }^{1}$ Graeme J Hankey, ${ }^{2}$ Sumathy Rangarajan, ${ }^{3}$ Siu Lim Chin, ${ }^{3}$ \\ Purnima Rao-Melacini, ${ }^{3}$ John Ferguson, ${ }^{4}$ Denis Xavier, ${ }^{5}$ Liu Lisheng, ${ }^{6,7}$ Hongye Zhang, $^{7}$ \\ Prem Pais, ${ }^{8}$ Patricio Lopez-Jaramillo, ${ }^{9}$ Albertino Damasceno, ${ }^{10}$ Peter Langhorne, ${ }^{11}$ \\ Annika Rosengren, ${ }^{12}$ Antonio L Dans, ${ }^{13}$ Ahmed Elsayed, ${ }^{14}$ Alvaro Avezum, ${ }^{15}$ \\ Charles Mondo, ${ }^{16}$ Andrew Smyth, ${ }^{4}$ Conor Judge (D) , ${ }^{17}$ Hans-Christoph Diener, ${ }^{18}$ \\ Danuta Ryglewicz, ${ }^{19}$ Anna Czlonkowska (D) , ${ }^{19}$ Nana Pogosova, ${ }^{20}$ \\ Christian Weimar, ${ }^{21,22}$ Romana Iqbal, ${ }^{23}$ Rafael Diaz, ${ }^{24}$ Khalid Yusoff, $^{25}$ \\ Afzalhussein Yusufali, ${ }^{26}$ Aytekin Oguz, ${ }^{27}$ Xingyu Wang, ${ }^{28}$ Ernesto Penaherrera, ${ }^{29}$ \\ Fernando Lanas (D) ${ }^{30}$ Okechukwu Samuel Ogah, ${ }^{31}$ Adensola Ogunniyi, ${ }^{32}$ \\ Helle K Iversen (D) ,' ${ }^{33}$ German Malaga, ${ }^{34}$ Zvonko Rumboldt, ${ }^{35}$ Shahram Oveisgharan, ${ }^{36}$ \\ Fawaz AlHussain, ${ }^{37}$ Magazi Daliwonga, ${ }^{38}$ Yongchai Nilanont, ${ }^{39}$ Salim Yusuf $^{3}$
}

\begin{abstract}
- Additional material is published online only. To view please visit the journal online (http://dx.doi.org/10.1136/ heartjnl-2019-316515).

For numbered affiliations see end of article.
\end{abstract}

\section{Correspondence to} Dr Martin O' Donnell, Clinical Research Facility, National University of Ireland Galway, Galway, Ireland; martin.odonnell@nuigalway.ie

Received 11 May 2020 Revised 27 August 2020 Accepted 10 September 2020 Published Online First 14 December 2020

\section{Linked}

- http://dx.doi.org/10.1136/ heartjnl-2020-318019

Check for updates

(C) Author(s) (or their employer(s)) 2021. No commercial re-use. See rights and permissions. Published by BMJ.

\section{To cite: $O^{\prime}$}

Donnell M, Hankey GJ,

Rangarajan $\mathrm{S}$, et al. Heart

2021:107:282-289.

\section{ABSTRACT}

Objective Hypertension is the most important modifiable risk factor for stroke globally. We hypothesised that country-income level variations in knowledge, detection and treatment of hypertension may contribute to variations in the association of blood pressure with stroke.

Methods We undertook a standardised case-control study in 32 countries (INTERSTROKE). Cases were patients with acute first stroke ( $n=13462)$ who were matched by age, sex and site to controls $(n=13$ 483). We evaluated the associations of knowledge, awareness and treatment of hypertension with risk of stroke and its subtypes and whether this varied by gross national income (GNI) of country. We estimated OR and population attributable risk (PAR) associated with treated and untreated hypertension.

Results Hypertension was associated with a graded increase in $\mathrm{OR}$ by reducing $\mathrm{GNI}$, ranging from $\mathrm{OR}$ 1.92 (99\% Cl 1.48 to 2.49 ) to OR 3.27 (2.72 to 3.93) for highest to lowest country-level GNI ( $p$ heterogeneity<0.0001). Untreated hypertension was associated with a higher OR for stroke (OR 5.25; 4.53 to 6.10 ) than treated hypertension (OR 2.60; 2.32 to 2.91 ) and younger age of first stroke (61.4 vs 65.4 years; $p<0.01)$. Untreated hypertension was associated with a greater risk of intracerebral haemorrhage (OR 6.95; 5.61 to 8.60 ) than ischaemic stroke (OR 4.76; 3.99 to 5.68 ). The PAR associated with untreated hypertension was higher in lowerincome regions, PAR $36.3 \%, 26.3 \%, 19.8 \%$ to $10.4 \%$ by increasing GNI of countries. Lifetime nonmeasurement of blood pressure was associated with stroke (OR 1.80; 1.32 to 2.46).

Conclusions Deficits in knowledge, detection and treatment of hypertension contribute to higher risk of stroke, younger age of onset and larger proportion of intracerebral haemorrhage in lower-income countries.

\section{INTRODUCTION}

Hypertension is an important modifiable ${ }^{1}$ risk factor for stroke. ${ }^{2-5} \mathrm{~A}$ number of international studies have reported regional and country income-level variations in awareness, treatment and control of hypertension. ${ }^{6-11}$ It is suspected that these variations in care gaps may contribute to differences in the risk, burden and pattern of strokes in countries at different economic levels. ${ }^{12} 13$ We explored whether variations in knowledge, detection (ie, awareness) and treatment of hypertension contribute to variations between countries at different economic levels in the magnitude of risk, burden and pattern of stroke and its subtypes, ischaemic and haemorrhagic stroke. Such information can assist in developing strategies to control hypertension and estimating the expected gains from improvements in hypertension detection and treatment globally and within countries of different income levels. ${ }^{14}$

\section{METHODS}

INTERSTROKE is a large international case control study ${ }^{315}$ conducted from March 2007 to September 2015 in 142 centres from 32 countries.

\section{Ethics}

All participants, or their proxy, provided informed written consent before taking part in the study.

\section{Participants}

Cases

Cases were patients with first stroke admitted to hospitals within the preceding 72 hours, and with recent symptoms onset ( $<5$ days), in whom neuroimaging by CT or MRI brain imaging could be completed within 1 week of presentation. Stroke was defined using the WHO clinical criteria for stroke. ${ }^{16}$ Neuroimaging was completed in $99.9 \%$ 
of cases. For patients unable to communicate adequately, proxy respondents were used. Key exclusions were: (1) patients unable to communicate because of severe stroke, aphasia or dementia who did not have a valid proxy respondent; (2) non-vascular causes; (3) current hospitalisation for acute coronary syndromes; 4) unable to get consent from proxy (online supplemental table 1).

\section{Controls}

Controls were either community-based $(54.7 \%)$ or hospitalbased (45.3\%). Hospital-based sources included patients admitted to hospital or attending outpatients for conditions or procedures not related to stroke or TIA or visitors or relatives of other in-patients. Detailed inclusion and exclusion criteria have been published ${ }^{15}$ (online supplemental table 2). Each control was matched for sex, age ( \pm 5 years) and site with cases, although this was extended to \pm 10 years for participants over 90 years of age. For the current analyses, cases and controls were matched for region and sex in all instances and age $195 \%$ within 5 years and $99.9 \%$ within 10 years).

\section{Measurement of risk factors}

Structured questionnaires were administered, and physical examinations were undertaken, in the same manner in cases and controls. Key vascular risk factors were measured in a manner consistent with the INTERHEART study ${ }^{17}$ (online supplemental appendix).

\section{Questionnaire specific to blood pressure}

Awareness of hypertension as a risk factor for stroke was recorded by asking all cases and controls whether they endorsed 'high blood pressure' to be 'associated with an increased risk of stroke'. A self-reported history of hypertension was based on a direct question to patient (and/or proxy) about whether they were diagnosed with hypertension. Preadmission antihypertensive therapy was captured from the medical notes and at interview from cases and controls. During the study, we implemented supplemental questions, completed by 6143 participants, which asked participants whether they ever had a prior blood pressure measurement. Hypertension was defined as self-reported history of hypertension or the composite of self-reported hypertension or blood pressure $\geq 140 / 90 \mathrm{~mm} \mathrm{Hg}$. For cases and controls, we used blood pressure at the time of interview, which was completed by research staff in all centres. Blood pressure is cases was adjusted to account for acute phase effect and described in the online supplemental appendix. Categorisation of economic level of country was based on information from World Bank in $2010{ }^{18}$ As the largest proportion of countries belonged to the upper middle-income category, we divided this category into two groups separated at the mid-point of gross national income (GNI), with four categories of high-income countries (HIC), upper middle-income countries-1 (UMIC-1), upper middleincome countries-2 (UMIC-2) and lower middle and lowerincome countries (LMIC/LICs), using GNI (online supplemental table 3).

\section{Statistical analysis}

Means and medians were calculated to summarise continuous variables and were compared by $t$ tests or appropriate nonparametric tests. We evaluated the association of blood pressure with stroke risk, and the association of knowledge of hypertension, awareness of hypertension and treatment of hypertension with stroke risk (all stroke, ischaemic stroke and intracerebral haemorrhage) and by income-status of country. We also evaluated the association of hypertension awareness with prior blood pressure measurement and pre-admission treatment for hypertension.

We used conditional logistic regression for stroke outcomes and unconditional logistic regression for outcomes related to hypertension. In addition to the variables being matched within a stratum, all models were additionally adjusted for exact age, smoking, diabetes mellitus, physical activity, diet, psychosocial factors, abdominal obesity, alcohol, cardiac risk factors and apolipoproteins. We estimated population attributable risk (PAR) using the method described by Bruzzi. ${ }^{19}$ For variables with more than two categories (eg, treated and untreated hypertension), we used partitioned PAR estimators, such that the PARs for differing categories sum to the total PAR for hypertension. We estimated income-category variations in the PAR associated with treated and untreated hypertension. We also modelled the expected reduction in proportion of stroke globally, and by income status category, in different scenarios of identification and screening of known hypertension, using GIF estimators ${ }^{20}$ which provide an estimate of the proportion of stroke that would be reduced if a risk factor was modified, based on summary estimates of association of treated and untreated hypertension with stroke risk in the current analyses. Impact fractions can be considered generalised versions of PAR percentages. Whereas a PAR percentage measures the proportional change in disease prevalence assuming a risk factor could be eliminated, impact fractions instead consider the effect of an intervention that causes a shift in the distribution of the risk factor, rather than a complete elimination. CI calculations (99\%) were based on standard Wald type intervals for OR, PAR and impact fractions, with the SE of the PAR and impact fraction estimators being estimated via Bootstrap. Bootstrapping was implemented by resampling $\mathrm{N}$ matched case/control pairs from the overall dataset with replacement 100 times, when the original dataset consisted of $\mathrm{N}$ matched pairs. Tests of interaction were calculated using the likelihood ratio method, comparing likelihoods of models with and without the interaction term of interest. Statistical analyses and graphics were produced with SAS V.9.4 for UNIX (SAS, Cary, North Carolina, USA) and the open source language R, V.3.4.2.

\section{RESULTS}

From March 2007 to September 2015, a total of 26945 participants were recruited from 32 countries, comprising 13462 individuals with acute first-ever stroke (cases) and 13483 controls. The mean age was 61.7 years (SD 13.4), ischaemic stroke in 10405 individuals $(77.3 \%)$ and intracerebral haemorrhage in 3057 (22.7\%). Table 1 and figure 1 describes the characteristics of cases and controls, for mean blood pressure, self-reported history of hypertension, use of antihypertensive therapy, knowledge that high blood pressure is a risk factor for stroke, prior blood pressure measurement and by income-level category of countries. The proportion of stroke due to intracerebral haemorrhage increased with reducing GNI of countries.

Lack of knowledge that hypertension causes stroke was associated with lower rates of any lifetime blood pressure measurement (OR 1.86; 99\% CI 1.47 to 2.37) and non-use of antihypertensive therapy in those with known hypertension (OR 1.60; 99\% CI 1.40 to 1.83), but was not an independent risk factor for stroke (OR 1.06; 0.85-1.33 overall, and OR 1.14; 0.94-1.38 for analysis confined to cases with patient respondent only). Lack of knowledge of hypertension as a risk factor for stroke was higher 


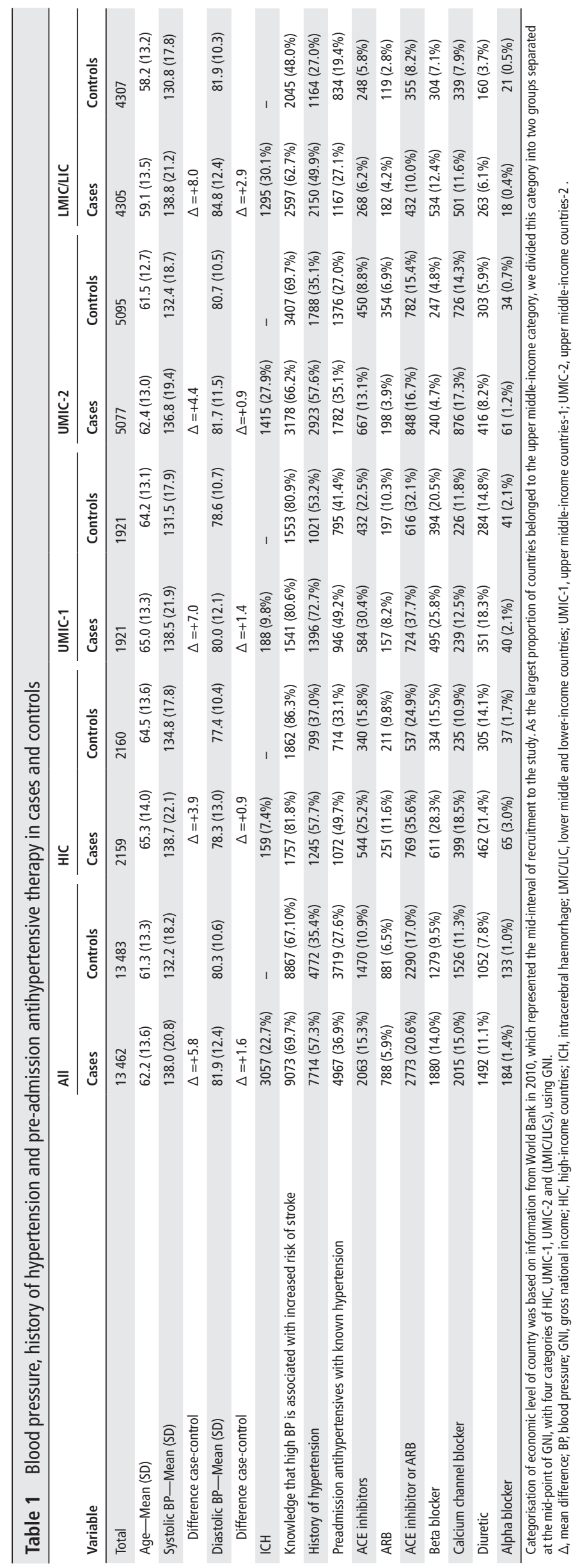




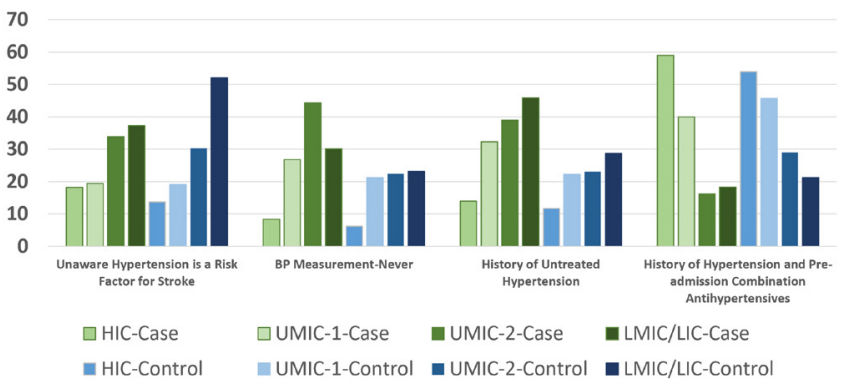

Figure 1 From left to right, figure reports proportion of cases and control that did not identify hypertension as a risk factor for stroke, proportion reporting no prior blood pressure measurement in their lifetime (subset of 6143 participants), proportion of cases and controls with a self-reported history of hypertension but not receiving preadmission antihypertensive therapy and proportion with a self-reported history of hypertension and receiving combination antihypertensive therapy. Income status of country based on gross national income. BP, blood pressure; HIC, high-income countries; LIC, lower-income countries; LMIC, lower middle-income countries.

in cases with intracerebral haemorrhage (34.1\%) than ischaemic stroke (29.2\%) and higher in LMIC/LICs than HICsfigure 1).

Of those completing supplementary questionnaire, 21.2\% of participants reported no prior blood pressure measurement, which was a risk factor for stroke (OR 1.80; 99\% CI 1.32 to 2.46), with non-measurement rates higher by lower-income level (figure 1). No prior measurement of blood pressure was more common in patients with intracerebral haemorrhage $(33.5 \%)$ than ischaemic stroke $(23.6 \%)(\mathrm{p}<0.01)$.

Hypertension was associated with stroke in all regions, with a graded increase in OR from high to lower-income countries, (OR 1.92; 99\% CI 1.48 to 2.49 for HIC, OR 2.29; $99 \%$ CI 1.73 to 3.02 ; for UMIC-1, OR 2.76; 99\% CI 2.40 to 3.18 for UMIC-2 and OR 3.27; 99\% CI 2.72 to 3.93 for LMIC/LICs; p-heterogeneity $<0.0001)$. Among participants with a selfreported history of hypertension (table 1), preadmission antihypertensive therapy use was lower in cases (64.4\%) than controls $(78.0 \%)(\mathrm{p}<0.001)$, with a gradient by income status of country, reflecting a treatment gap even among those who were aware of their hypertension (table 1, figure 1). Categorising those with a self-reported history of hypertension into those receiving, and not receiving preadmission antihypertensive therapy, revealed a stronger magnitude of association between prior history of hypertension without antihypertensive therapy (OR 5.25; $4.53-$ 6.10) than those who reported pre-admission antihypertensive therapy (OR 2.60; 2.32-2.91) (P-interaction=0.01) (table 2, online supplemental table 4). Untreated hypertension was associated with a greater risk of intracerebral haemorrhage (OR 6.95; 5.61-8.60) than ischaemic stroke (OR 4.76; 3.99 to 5.68).

Figure 2 reports the PAR by income-level category, divided into those who were unaware of hypertension (no prior diagnosis), aware of hypertension but untreated and aware of their hypertension and treated, demonstrating a graded increase in the proportion of the PAR associated with untreated hypertension, by lower-income country category. While the prevalence of hypertension was lower in lower-income countries (compared with higher), the greater OR associated with untreated hypertension, resulted in similar PARs by income group. The PAR due to untreated hypertension (both among those detected and those undetected) was $10.4 \%$ in HICs, $19.8 \%$ in UMIC-1, $26.3 \%$ in UMIC-2 and $36.3 \%$ in LMIC/LICs ( $<<0.001)$. An analysis restricted to community-based controls demonstrated consistent findings (online supplemental figure 1). Online supplemental figure 2 report partitioned PAR for hypertension by age, sex and type of stroke (ischaemic and intracerebral haemorrhage). Use of antihypertensive therapy was lower in younger age groups, and the increased magnitude of association between hypertension and stroke in younger age groups persisted, after stratification for use of antihypertensive therapy (figure 3). Mean age of stroke was 65.4 years in those with treated hypertension and 61.4 years in those with untreated hypertension $(\mathrm{p}<0.01)$.

Table 3 reports the association of hypertension with stroke, by number of preadmission antihypertensive medications reported. Use of combination antihypertensive therapy (two or more agents) among those with hypertension was $28.9 \%$ overall, $51.9 \%$ in HICs, $40.7 \%$ in UMIC-1, 20.7\% in UMIC-2 and $17.9 \%$ in LICs. The risk association with treated hypertension was OR 2.91 (2.56-3.31) for one agent and OR 2.16 (1.86 to 2.50) for two agents or more. Non-use of antihypertensive therapy was associated with a higher OR in younger (OR $7.85 ; 5.84$ to 10.55 ) than older age (OR $4.26 ; 3.54$ to 5.12 for those $>55$ years). (P-interaction $<0.01)$. Use of antihypertensive therapy and combination therapy was lower in lower-income regions (figure 1). Figure 3 reports the association of hypertension by age categories and

Table 2 Association between history of hypertension and stroke, by preadmission use of antihypertensive therapy

\begin{tabular}{|c|c|c|c|c|c|}
\hline & All & HIC & UMIC-1 & UMIC-2 & LMIC/LIC \\
\hline & OR $(99 \% \mathrm{Cl})$ & OR $(99 \% \mathrm{CI})$ & OR $(99 \% \mathrm{Cl})$ & OR $(99 \% \mathrm{Cl})$ & OR $(99 \% \mathrm{CI})$ \\
\hline \multicolumn{6}{|l|}{ All stroke } \\
\hline Treated hypertension & 2.60 (2.32 to 2.91$)$ & 2.22 (1.66 to 2.98$)$ & 2.01 (1.48 to 2.74 ) & 2.59 (2.19 to 3.06$)$ & 3.68 (2.85 to 4.76$)$ \\
\hline \multicolumn{6}{|l|}{ Ischaemic stroke } \\
\hline Untreated hypertension & 4.76 (3.99 to 5.68 ) & 2.78 (1.61 to 4.81$)$ & 4.12 (2.63 to 6.44$)$ & 4.54 (3.50 to 5.90$)$ & 6.80 (4.72 to 9.80$)$ \\
\hline Untreated hypertension & 6.95 (5.61 to 8.60$)$ & 4.44 (1.19 to 16.58$)$ & 4.33 (1.91 to 9.84 ) & 7.76 (5.75 to 10.47$)$ & 7.66 (5.32 to 11.03 ) \\
\hline Treated hypertension & 2.77 (2.30 to 3.32 ) & 1.63 (0.74 to 3.57 ) & 2.20 (1.05 to 4.64$)$ & 3.02 (2.36 to 3.87 ) & 3.04 (2.14 to 4.30$)$ \\
\hline
\end{tabular}

Categorisation of economic level of country was based on information from World Bank in 2010, which represented the mid-interval of recruitment to the study. As the largest proportion of countries belonged to the upper middle-income category, we divided this category into two groups separated at the mid-point of GNI, with four categories of HIC, UMIC-1, UMIC-2 and LMIC/LICS, using GNI. Multivariable model including, age, smoking, WHR, diabetes, physical activity, alternate healthy eating index, alcohol intake, psychosocial factors, apolipoproteins and cardiac risk factors. Reference category is individuals with no prior hypertension or hypertension on interview blood pressure. GNI, gross national income; HIC, high-income countries; LMIC/LIC, lower middle and lower-income countries; UMIC-1, upper middle-income countries-1; UMIC-2, upper middleincome countries-2. 


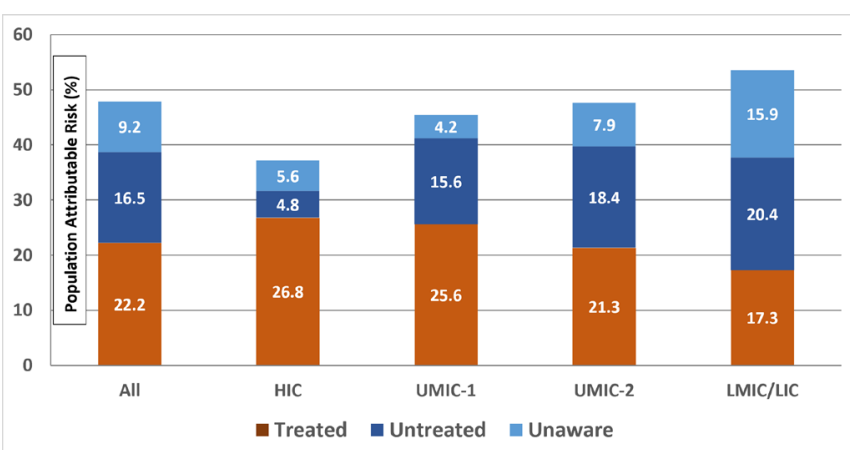

Figure 2 Figure reports partitioned PAR, by awareness and treatment status (ie, subdivided into proportion due to treated hypertension, known hypertension but untreated and undiagnosed hypertension). Data are shown overall and in GNI-level groups. Figure demonstrates a reduction in proportion of PAR associated with treated hypertension by income-category, with highest PAR for treated hypertension in $\mathrm{HIC}$, and an increase in proportion of PAR associated with untreated hypertension in lower-income countries. Multivariable model including age, smoking, waist-to-hip ratio (WHR), diabetes, physical activity, alternate healthy eating index, alcohol intake, psychosocial factors, apolipoproteins and cardiac risk factors. GNI, gross national income; HIC, high-income countries; LIC, lower-income countries; LMIC, lower middle-income countries; PAR, population attributable risk.

GNI, for untreated hypertension and treated hypertension (with one or combination antihypertensive therapy). Both younger age and lower GNI are associated with higher ORs for the association of hypertension with stroke, which is attenuated with antihypertensive use. We modelled anticipated gains from improved treatment of known hypertension, using an estimate of generalised impact fraction of two scenarios. First, use of antihypertensive drugs at currently observed intensity in those known to have hypertension indicates that $10.3 \%$ of stroke globally would be avoided if known hypertensives were treated, but not necessarily controlled. This would be $3.8 \%(2.7 \%-5.0 \%)$ avoided in
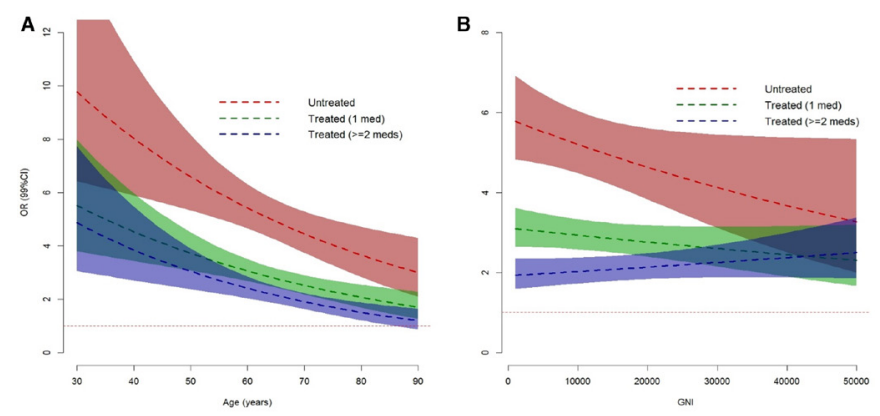

Figure 3 Figures report the association of hypertension with stroke by age (A) and GNI (B), demonstrating an increased slope in magnitude of association of hypertension with stroke by reducing age and reducing $\mathrm{GNI}$, which is modified by treatment status. Within increasing intensity of antihypertensive therapy, there is a diminution in slope of curve. While a gradient remains for risk of stroke by age among treatment groups, there is an inversion of gradient by GNI. These figures illustrate that increased uptake of antihypertensive therapy are expected to have greatest impact in younger populations and in lower-income regions. Multivariable model including age, smoking, WHR, diabetes, physical activity, alternate healthy eating index, alcohol intake, psychosocial factors, apolipoproteins and cardiac risk factors. GNI, gross national income; PAR, population attributable risk.
HIC, 10.4\% (8.1\%-12.7\%) in UMIC-1, 11.5\% (9.5\%-13.5\%) in UMIC-2 and $12.0 \%(9.6 \%-14.5 \%)$ in LMIC/LICs. Second is use of at least two antihypertensive agents in all known hypertensives; this indicates that $17.6 \%(13.6 \%-21.6 \%)$ of stroke globally would be eliminated, $9.1 \%(6.0 \%-12.1 \%)$ in HICs, $17.8 \%$ (12.9\%-22.6\%) in UMIC-1, 20.1\% (15.1\%-25.1\%) in UMIC-2 and $18.4 \%(14.6 \%-22.1 \%)$ in LMIC/LICs. The GIF was larger for intracerebral haemorrhage $(32.4 \%)$ than ischaemic stroke (13.5\%), reflecting the higher prevalence of hypertension and larger OR for untreated compared with treated hypertension for intracerebral haemorrhage compared with ischaemic stroke.

\section{DISCUSSION}

Hypertension is a key modifiable risk factor for stroke in all regions of the world and for both primary stroke subtypes. Poor knowledge that hypertension is a risk factor for stroke is associated with lower awareness of hypertension and non-use of antihypertensive therapy. Each of these care gaps was more common in lower-income countries, compared with higher-income countries, resulting in a higher OR associated with hypertension in lower-income countries. Hypertension, and particularly untreated hypertension, is a stronger risk factor for intracerebral haemorrhage than ischaemic stroke. Untreated hypertension, more common in lower-income countries, is associated with younger age of first stroke.

The PURE study reported major gaps in detection $(46.5 \%$ of participants with hypertension were aware they had hypertension) and control (32.5\%) of hypertension globally, with larger care gaps in lower-income countries, compared with higher income countries,. ${ }^{6}$ Our findings, in the current analysis of the INTERSTROKE study, extend these observations by quantifying the contribution of these gaps to the global burden, and epidemiologic pattern, of stroke. While we report a graded increase in the OR for hypertension associated with stroke by lower-income status of country, which resulted in a larger PAR for a given prevalence of hypertension, this was mostly related to a larger proportion of untreated hypertension in lower-income countries. Untreated hypertension was more strongly associated with intracerebral haemorrhage (versus ischaemic stroke) and predicted a younger age of stroke onset, meaning that care gaps in detection and treatment of hypertension result in a larger burden of stroke and differences in case mix, case-fatality (due to higher intracerebral haemorrhage) and socioeconomic consequences due to younger age of onset, which are observations consistent with other epidemiologic studies. ${ }^{25}$ Our data also suggest that greater population-level use of antihypertensive therapy is now favourably changing the epidemiologic association between blood pressure and stroke, whereby countries with higher use of antihypertensive therapies have a lower risk of stroke associated with hypertension, lower proportion of intracerebral haemorrhage and older age of first stroke onset.

A limitation of the PAR estimate is that it provides a theoretical estimate of the reduction in stroke associated with the complete elimination of hypertension (which is not ever likely), rather than an estimate of anticipated gains from available and potentially feasible interventions. Of greater relevance to health policy, therefore, is the anticipated gains with greater detection and control of hypertension. The impact fraction associated with treatment of hypertension is lower than the corresponding PAR. This difference between PAR and impact fraction likely reflects a number of factors. We report a residual risk associated with controlled hypertension, which is consistent with prior studies reporting have reported higher cardiovascular risk 
Table 3 Association between history of hypertension and stroke, by number of antihypertensive agents preadmission

\begin{tabular}{|c|c|c|c|c|c|c|}
\hline \multirow[b]{2}{*}{ Overall } & \multicolumn{2}{|l|}{ All stroke } & \multicolumn{2}{|l|}{ Ischaemic stroke } & \multicolumn{2}{|c|}{ Intracerebral haemorrhage } \\
\hline & OR $(99 \% \mathrm{Cl})$ & PAR $(99 \% \mathrm{Cl})$ & OR $(99 \% \mathrm{Cl})$ & PAR $(99 \% \mathrm{Cl})$ & OR $(99 \% \mathrm{Cl})$ & PAR $(99 \% \mathrm{Cl})$ \\
\hline No antihypertensive & 5.26 (4.53 to 6.11$)$ & $16.5 \%(15.3$ to 17.7$)$ & 4.77 (4.00 to 5.69$)$ & $14.3 \%(13.0$ to 15.7$)$ & 6.96 (5.62 to 8.62 ) & $24.1 \%$ (16.8 to 31.4$)$ \\
\hline 1 Agent used & 2.91 (2.56 to 3.31$)$ & $14.1 \%$ (12.7 to 15.4$)$ & $2.93(2.52$ to 3.41$)$ & $13.9 \%$ (12.1 to 15.7$)$ & 3.38 (2.77 to 4.12$)$ & $15.9 \%$ (8.6 to 23.2 ) \\
\hline$\geq 2$ Agents used & 2.16 (1.86 to 2.50$)$ & $7.9 \%(6.4$ to 9.3$)$ & 2.35 (1.99 to 2.78) & $9.6 \%$ (8.0 to 11.2 ) & $1.70(1.30$ to 2.24$)$ & $3.2 \%(-9.4$ to 15.9$)$ \\
\hline
\end{tabular}

Multivariable model including age, smoking, WHR, diabetes, physical activity, alternate healthy eating index, alcohol intake, psychosocial factors, apolipoproteins and cardiac risk factors. Reference category is individuals with no prior hypertension or hypertension on interview blood pressure.

PAR, population attributable risk.

among individuals with controlled hypertension, compared with those without hypertension. ${ }^{21} 22$ This residual risk of stroke may also be due to concomitant risk factors, suboptimal treatment of hypertension, risk associated with intermediate phenotype (eg, small vessel disease) related to a period of undiagnosed hypertension or unmeasured confounders such as undetected atrial fibrillation. This implies that strategies to reduce the risk of stroke in those with hypertension needs to control blood pressure and include combined approaches such as lipid lowering, ${ }^{23}$ smoking cessation and detection and treatment of atrial fibrillation. ${ }^{24}$ Such a combined approach is more important for prevention of ischaemic stroke, compared with intracerebral haemorrhage where the impact fraction associated with hypertension is $32.4 \%$ for treating known hypertension (compared with 13.4\% for ischaemic stroke) due to a larger OR related to untreated hypertension and greater relative risk reduction with hypertension control. ${ }^{25}$ In addition, our estimates of impact fractions are based on the current INTERSTROKE population, rather than population-wide efforts to prevent hypertension derived from international prospective cohort studies.

Most individuals with hypertension require combination therapy to control blood pressure. ${ }^{26}$ We observed the lowest risk of stroke associated with hypertension in the subgroup receiving two or more antihypertensive agents, particularly for intracerebral haemorrhage (table 3). While we report a graded reduction in use of combination antihypertensive regimens by lowerincome-level groups, all had low rates of combination antihypertensive use, including HICs, which is also consistent with studies reporting high rates of treatment but low rates of hypertension control in HICs. ${ }^{34}$ We observed a sequential lowering of stroke risk association with increased treatment intensity of hypertension. Interventions that target barriers to optimal detection and treatment of hypertension, including educational programmes to increase awareness of hypertension, non-physician led screening and algorithm-based use of fixed-dose combination therapy (eg, Polycap, ${ }^{27}$ low-cost generic medications, have the potential to exact major gains in reducing stroke. ${ }^{28-30}$

Our study has some limitations. The case control nature of our study incurs unavoidable sources of bias, such as recall bias. For example, the prevalence of knowledge of hypertension as a risk factor for stroke among stroke patients may not reflect their knowledge prior to their hospital admission, which is expected to affect knowledge among cases more than controls, and which may account for a similar rate among cases and controls, despite lower rates of detection and treatment in cases. Prior assessment of blood pressure measurement was higher in controls than reported in the PURE study and other studies. ${ }^{6-8}$ This difference may be due to varying approaches to selecting controls from outpatient or general practice settings, where measurement of blood pressure may be routine, and the inclusion of a larger proportion of urban centres, as we required hospital sites to have access to neuroimaging. Additionally, the rates of treatment for hypertension among controls were also higher, but more consistent with estimates reported in the PURE study, than estimates for undetected hypertension. ${ }^{6}$ As such, our estimates for the PAR related to undetected hypertension likely represents an underestimate, but our estimates of PAR and impact fraction related to uptake of antihypertensive treatment among those with known hypertension are expected to be more reliable. Our approach to adjusting for the acute phase effect of stroke on blood pressure may have overpenalised, especially for intracerebral haemorrhage (online supplemental figures 2 and 3 ) and may have resulted in underestimating the frequency of undiagnosed and uncontrolled hypertension, but the direction of bias would most likely result in an underestimate of the PAR related to undiagnosed (and controlled) hypertension, and therefore, our estimates should be considered conservative. For this reason, we did not report an estimate for impact fraction for prior undiagnosed hypertension.

\section{Key messages}

\section{What is already known on this subject?}

- Hypertension is the leading modifiable risk factor for stroke globally. Rates of detection, treatment and control are inadequate in all regions of the world, but particularly in lowincome and middle-income countries.

\section{What might this study add?}

- In this paper, we report that deficits in knowledge, detection and treatment of hypertension contribute to higher risk of stroke, younger age of onset and larger proportion of intracerebral haemorrhage in lower-income countries (compared with higher income regions). Alternatively viewed, our data suggest that greater population-level use of antihypertensive therapy is now favourably changing the epidemiologic association between blood pressure and stroke, whereby countries with higher use of antihypertensive therapies have a lower risk of stroke associated with hypertension, lower proportion of intracerebral haemorrhage and older age of first stroke onset.

\section{How might this impact on clinical practice?}

- These data suggest that strategies to improve knowledge about hypertension in the population, access to blood pressure screening programmes and low-cost combination antihypertensive therapy could have a major impact on reducing the global burden of stroke, particularly premature stroke in lower middle-income countries. 


\section{SUMMARY/CONCLUSION}

In conclusion, deficits in knowledge, awareness and treatment of hypertension contribute to higher risk of stroke, younger age of onset and larger proportion of intracerebral haemorrhage in lower-income countries, compared with HICs. These data suggest that strategies to improve knowledge about hypertension in the population, access to blood pressure screening programmes and low-cost combination antihypertensive therapy could have a major impact on reducing the global burden of stroke, particularly premature stroke in LMICs.

\section{Author affiliations}

'HRB-Clinical Research Facility, National University of Ireland Galway, Galway, Ireland

${ }^{2}$ Medical School, Faculty of Health and Medical Sciences, University of Western Australia, Perth, Western Australia, Australia

${ }^{3}$ Dept of Medicine, Population Health Research Institute, McMaster University and

Hamilton Health Sciences, Hamilton, Ontario, Canada

${ }^{4}$ National University of Ireland Galway, Galway, Ireland

${ }^{5}$ St John's Research Insitiute, Bangalore, Karnataka, India

${ }^{6}$ National Center for Cardiovascular Diseases China, Xicheng District, Beijing, China

${ }^{7}$ Beijing Hypertension League Institute, Beijing, China

${ }^{8}$ St John's National Academy of Health Sciences, Bangalore, Karnataka, India

${ }^{9}$ Instituto de Investigaciones MASIRA, Universidad de Santander, Bucaramanga, Colombia

${ }^{10}$ Faculty of Medicine, Eduardo Mondlane University, Maputo, Mozambique

${ }^{11}$ Academic Section of Geriatric Medicine, Glasgow Royal Infirmary, University of Glasgow, Glasgow, UK

${ }^{12}$ Department of Molecular and Clinical Medicine, University of Gothenburg and Region Västra Götaland, Sahlgrenska University Hospital, Goteborg, Sweden

${ }^{13}$ University of the Philippines Manila College of Medicine, Manila, Metro Manila, Philippines

${ }^{14}$ Alzaiem Alazhari University, Khartoum, Sudan

${ }^{15}$ International Research Center, Hospital Alemão Oswaldo Cruz, São Paulo, Brazil

${ }^{16}$ Kiruddu National Referral Hospital, Kampala, Uganda

${ }^{17}$ Medicine, National University of Ireland Galway, Galway, Galway, Ireland

${ }^{18}$ Department of Neurology, University Hospital Essen, Essen, Nordrhein-Westfalen, Germany

${ }^{19}$ Military Institute of Aviation Medicine, Warsaw, Poland

${ }^{20}$ National Medical Research Center of Cardiology, Moscow, Russia, Moskva, Russian Federation

${ }^{21}$ Neurology, Institute for Medical Informatics, Biometry and Epidemiology, University of Duisburg-Essen, Essen, Germany

${ }^{22} \mathrm{BDH}$-clinic Elzach, Elzach, Germany

${ }^{23}$ Department of Community Health Sciences and Medicine, Aga Khan University, Karachi, Pakistan

${ }^{24}$ Estudios Clínicos Latino America (ECLA), Instituto Cardiovascular de Rosario (ICR), Rosario, Argentina

${ }^{25}$ Universiti Teknologi MARA, Selayang, Selangor and UCSI University, Kuala Lumpur, Malaysia

${ }^{26}$ Hatta Hospital, Dubai Health Authority/Dubai Medical College, Dubai, UAE

${ }^{27}$ Department of Internal Medicine, Istanbul Medeniyet University, Istanbul, Istanbul, Turkey

${ }^{28}$ Laboratory of Human Genetics, Beijing Hypertension League Institute, Beijing, China

${ }^{29}$ Department of Cardiology, Luis Vernaza General Hospital, Guayaquil, Guayas,

Ecuador

${ }^{30}$ Internal Medicine, Universidad de La Frontera, Temuco, Chile

${ }^{31}$ Division of Cardiology, University of Ibadan, Ibadan, Oyo, Nigeria

${ }^{32}$ Neurology Unit, Department of Medicine, University College Hospital Ibadan, Ibadan, Oyo, Nigeria

${ }^{33}$ Department of Neurology, University of Copenhagen, Rigshospitalet, Denmark

${ }^{34}$ School of Medicine, Universidad Peruana Cayetano Heredia, Lima, Peru

${ }^{35}$ Department of Medicine, University of Split, Split, Splitsko-dalmatinska, Croatia

${ }^{36}$ Rush University Medical Center, Chicago, Illinois, USA

${ }^{37}$ King Saud University, Riyadh, Riyadh Province, Saudi Arabia

${ }^{38}$ Department of Medicine, University of Limpopo, Sovenga, Limpopo, South Africa

${ }^{39}$ Division of Neurology, Department of Medicine, Siriraj Hospital, Mahidol University, Bangkok, Thailand

Twitter Patricio Lopez-Jaramillo @Machachi2016 and Conor Judge @conorjudge

Contributors All authors contributed to the collection of data, discussions and interpretation of the data, and to the writing of the report. All authors reviewed and approved the manuscript for submission. The study was designed, the analyses were planned and the manuscript was drafted by MO'D and SY. Statistical analyses were performed by PR-M and JF. MO'D and SY wrote the first draft of the manuscript.

Funding The INTERSTROKE study is funded by the Canadian Institutes of Health Research, Heart and Stroke Foundation of Canada, Canadian Stroke Network, Swedish Research Council, Swedish Heart and Lung Foundation, The Health \& Medical Care Committee of the Regional Executive Board, Region Västra Götaland, and through unrestricted grants from several pharmaceutical companies with major contributions from Astra Zeneca, Boehringer Ingelheim (Canada), Pfizer (Canada), MERCK, Sharp and Dohme], Swedish Heart and Lung Foundation, UK Chest, and UK Heart and Stroke.

Disclaimer No medical writer or other people were involved in the design, analysis or writing of this manuscript.

Competing interests None declared.

\section{Patient consent for publication Not required.}

Ethics approval The study was approved by the ethics committees in all participating centres.

Provenance and peer review Not commissioned; externally peer reviewed. Data availability statement No additional data are available.

Supplemental material This content has been supplied by the author(s). It has not been vetted by BMJ Publishing Group Limited (BMJ) and may not have been peer-reviewed. Any opinions or recommendations discussed are solely those of the author(s) and are not endorsed by BMJ. BMJ disclaims all liability and responsibility arising from any reliance placed on the content. Where the content includes any translated material, BMJ does not warrant the accuracy and reliability of the translations (including but not limited to local regulations, clinical guidelines, terminology, drug names and drug dosages), and is not responsible for any error and/or omissions arising from translation and adaptation or otherwise.

\section{ORCID iDs}

Conor Judge http://orcid.org/0000-0001-9473-2920

Anna Czlonkowska http://orcid.org/0000-0002-1956-1866

Fernando Lanas http://orcid.org/0000-0003-3595-9759

Helle K Iversen http://orcid.org/0000-0002-5880-6352

\section{REFERENCES}

1 Ettehad D, Emdin CA, Kiran A, et al. Blood pressure lowering for prevention of cardiovascular disease and death: a systematic review and meta-analysis. Lancet 2016:387:957-67.

2 Feigin VL, Roth GA, Naghavi M, et al. Global burden of stroke and risk factors in 188 countries, during 1990-2013: a systematic analysis for the global burden of disease study 2013. Lancet Neurol 2016;15:913-24.

3 O'Donnell MJ, Chin SL, Rangarajan S, et al. Global and regional effects of potentially modifiable risk factors associated with acute stroke in 32 countries (INTERSTROKE): a case-control study. Lancet 2016;388:761-75.

4 Forouzanfar MH, Liu P, Roth GA, et al. Global burden of hypertension and systolic blood pressure of at least 110 to 115 MM Hg, 1990-2015. JAMA 2017;317:165-82.

5 Rapsomaniki E, Timmis A, George J, et al. Blood pressure and incidence of twelve cardiovascular diseases: lifetime risks, healthy life-years lost, and age-specific associations in 1.25 million people. Lancet 2014;383:1899-911.

6 Chow CK, Teo KK, Rangarajan S, et al. Prevalence, awareness, treatment, and control of hypertension in rural and urban communities in high-, middle-, and low-income countries. JAMA 2013:310:959-68.

7 Mills KT, Bundy JD, Kelly TN, et al. Global disparities of hypertension prevalence and control: a systematic analysis of population-based studies from 90 countries. Circulation 2016;134:441-50.

8 Melgarejo JD, Maestre GE, Thijs L, et al. Prevalence, treatment, and control rates of conventional and ambulatory hypertension across 10 populations in 3 continents. Hypertension 2017;70:50-8.

9 Lawes CMM, Vander Hoorn S, Rodgers A, et al. Global burden of blood-pressurerelated disease, 2001. Lancet 2008;371:1513-8.

10 Kearney PM, Whelton M, Reynolds K, et al. Global burden of hypertension: analysis of worldwide data. Lancet 2005;365:217-23.

11 Ikeda N, Sapienza D, Guerrero R, et al. Control of hypertension with medication: a comparative analysis of national surveys in 20 countries. Bull World Health Organ 2014:92:10-19.

12 Feigin VL, Norrving B, Mensah GA. Global burden of stroke. Circ Res 2017:120:439-48

13 Feigin VL, Mensah GA, Norrving B, et al. Atlas of the global burden of stroke (19902013): the GBD 2013 study. Neuroepidemiology 2015;45:230-6.

14 Feigin VL, Norrving B, George MG, et al. Prevention of stroke: a strategic global imperative. Nat Rev Neurol 2016;12:501-12. 
15 O'Donnell M, Xavier D, Diener C, et al. Rationale and design of INTERSTROKE: a global case-control study of risk factors for stroke. Neuroepidemiology 2010;35:36-44.

16 Hatano $S$. Experience from a multicentre stroke register: a preliminary report. Bull World Health Organ 1976;54:541-53.

17 Yusuf S, Hawken S, Ounpuu S, et al. Effect of potentially modifiable risk factors associated with myocardial infarction in 52 countries (the INTERHEART study): casecontrol study. Lancet 2004;364:937-52.

18 World Bank. Available: http://siteresources.worldbank.org/DATASTATISTICS/Resources/ GNI.pdf

19 Bruzzi P, Green SB, Byar DP, et al. Estimating the population attributable risk for multiple risk factors using case-control data. Am J Epidemiol 1985;122:904-14.

20 Barendregt JJ, Veerman JL. Categorical versus continuous risk factors and the calculation of potential impact fractions. J Epidemiol Community Health 2010:64:209-12.

21 Barengo NC, Hu G, Kastarinen M, et al. The effects of awareness, treatment and control of hypertension on future stroke incidence in a community-based population study in Finland. J Hypertens 2009;27:1459-65.

22 Lawlor DA, Kim L, Morris R, et al. Survival with treated and well-controlled blood pressure: findings from a prospective cohort study. PLoS One 2011;6:e17792.

23 Yusuf S, Lonn E, Pais P, et al. Blood-Pressure and cholesterol lowering in persons without cardiovascular disease. N Engl J Med 2016;374:2032-43.
24 Blacher J, Levy BI, Mourad J-J, et al. From epidemiological transition to modern cardiovascular epidemiology: hypertension in the 21st century. Lancet 2016;388:530-2.

25 Katsanos AH, Filippatou A, Manios E, et al. Blood pressure reduction and secondary stroke prevention: a systematic review and metaregression analysis of randomized clinical trials. Hypertension 2017;69:171-9.

26 Cushman WC, Ford CE, Cutler JA, et al. Success and predictors of blood pressure control in diverse North American settings: the antihypertensive and lipid-lowering treatment to prevent heart attack trial (ALLHAT). J Clin Hypertens 2002;4:393-404.

27 Yusuf S, Pais P, et al, Indian Polycap Study (TIPS). Effects of a polypill (Polycap) on risk factors in middle-aged individuals without cardiovascular disease (tips): a phase II, double-blind, randomised trial. Lancet 2009;373:1341-51.

28 He J, Irazola V, Mills KT, et al. Effect of a community health Worker-Led multicomponent intervention on blood pressure control in low-income patients in Argentina: a randomized clinical trial. JAMA 2017:318:1016-25.

29 Attaei MW, Khatib R, McKee M, et al. Availability and affordability of blood pressurelowering medicines and the effect on blood pressure control in high-income, middleincome, and low-income countries: an analysis of the pure study data. Lancet Public Health 2017:2:e411-9.

30 Xavier D, Gupta R, Kamath D, et al. Community health worker-based intervention for adherence to drugs and lifestyle change after acute coronary syndrome: a multicentre, open, randomised controlled trial. Lancet Diabetes Endocrinol 2016;4:244-53. 